\title{
Contact-dependent growth inhibition tRNase toxin-immunity protein complexes from Escherichia coli 3006 and Klebsiella pneumoniae 342
}

\author{
Karolina Michalska ${ }^{1}$, William H. Eschenfeldt ${ }^{1}$, Lucy Stols ${ }^{1}$, Grant C. Gucinski ${ }^{3}$, Celia W. \\ Goulding $^{4,5}$, Christopher S. Hayes ${ }^{3,6}$, Andrzej Joachimiak ${ }^{1,2,7}$ \\ ${ }^{1}$ Midwest Center for Structural Genomics ${ }^{2}$ Structural Biology Center, Biosciences Division, \\ Argonne National Laboratory, Lemont, IL 60439, USA (kmichalska@anl.gov) \\ ${ }^{3}$ Biomolecular Science and Engineering Program $/{ }^{6}$ Department of Molecular, Cellular and \\ Developmental Biology, University of California, Santa Barbara, Santa Barbara, CA 93106, USA \\ ${ }^{4}$ Department of Molecular Biology \& Biochemistry $/{ }^{5}$ Pharmaceutical Sciences, University of \\ California Irvine, Irvine, CA 92697, USA \\ ${ }^{7}$ Department of Biochemistry and Molecular Biology, University of Chicago, Chicago, IL 60637, \\ USA
}

Contact Dependent growth Inhibition (CDI) represents one means of interbacterial communication among kin cells, facilitating cooperative behaviors including biofilm formation, persistence and virulence. Mechanistically, it utilizes a two-partner secretion system whereby the filamentous CdiA protein is displayed on the cell surface. This allows delivery of the Cterminal toxin cargo (CdiA-CT) to neighboring cells upon contact. $\mathrm{CDI}^{+}$cells also produce Cdil immunity protein that specifically binds to CdiA-CT and confers protection from toxin activities. Some CDI toxins are tRNases that display similarity to the previously characterized toxins, colicins $D$ and $E 5$, belonging to a larger family of RNases known as BECR (barnase/EndoU/colicin E5-D/RelE). While these colicins cleave anticodon loops, the currently characterized CdiA tRNases remove $3^{\prime}$ end of acceptor arms, thereby exhibiting activity not previously reported for any RNase. Here we present two novel CdiA toxins with such a function. The crystal structures of CdiA-CT·Cdil complexes from Escherichia coli 3006 and Klebsiella pneumoniae 342 show CdiA-CT toxins with folds similar to other BECR members, despite the lack of detectable sequence similarity to those proteins. Though the toxins share significant structural homology and have conserved active sites, their cognate immunity proteins have completely unrelated structures and the two complexes assume distinct oligomeric states. The immunity proteins block toxin function by interacting with analogous CdiA-CT surfaces in the vicinity of active sites. 